\title{
Single-Molecule Luminescence Spectroscopy Induced by Local Electron Tunneling
}

\author{
Pierre Brodard*
}

\begin{abstract}
Chemical identification at the single-molecular scale requires high spatial resolution as well as rich intrinsic information on the nature of the sample. Fluorescence and phosphorescence spectra of individual fullerene molecules excited by the highly localized current of electrons in a scanning tunneling microscope junction are presented. Pure electronic as well as vibronically induced bands are assigned by comparison with non-local spectroscopy and results of quantum chemical calculations, leading to unambiguous chemical identification of $\mathrm{C}_{60}$ molecules on $\mathrm{NaCl}$-covered $\mathrm{Au}(111)$ substrates. Future developments are discussed.
\end{abstract}

Keywords: Fullerene nanocrystals · Scanning tunneling microscopy (STM) - Single-molecule spectroscopy · Tip-enhanced Raman spectroscopy (TERS) - Tip-induced luminescence

Optical spectroscopy, by probing the absorption or emission of photons by molecules, provides a wealth of information about the intrinsic nature of a sample, and is therefore one of the most widely used experimental techniques in chemistry. The absorption of ultraviolet or visible light (UV/VIS spectroscopy), which excites electronic transitions in the species, tells us about the energy levels, complemented by fluorescence measurements, probing the de-excitations between electronic states. At lower energies, infrared (IR) light excites vibrational transitions in the molecules. In Raman spectroscopy, visible light is scattered by non-absorbing samples as a result of inelastic collisions between photons and molecules, the former either losing energy when exciting vibrations in the latter (Stokes band) or retrieving the energy of thermally excited vibrations (anti-Stokes

\footnotetext{
${ }^{*}$ Correspondence: Dr. P. Brodard

Ecole Polytechnique Fédérale de Lausanne (EPFL) Institut de Physique des Nanostructures

Bâtiment BSP-CUB

$\mathrm{CH}-1015$ Lausanne

Tel.: +41216930449

Fax: +41216930422

E-Mail: pierre.brodard@epfl.ch
}

band). The vibrational spectrum is a unique signature of a species, and thus both IR and Raman spectroscopies allow unambiguous chemical identification.

All the above processes are sensitive to the environment. For example, the vibrational spectrum of a molecule in the gas phase is different from the response of the species adsorbed on a surface: the molecule-surface interaction restricts the symmetry of the compound, thus modifying the selection rules. It is therefore very appealing to use optical spectroscopy to investigate nanoscale structures in order to extract local, single-molecule information in various environments. However, classic optical spectroscopy intrinsically lacks spatial resolution. In far-field techniques the resolution $f$ indeed cannot be better than several hundreds of nanometers due to the diffraction limit (Eqn. (1)) with visible light of wavelength $\lambda$. This is several orders of magnitude larger than molecules, carbon-carbon bond lengths being for instance of the order of hundreds of picometers.

$$
f^{\text {diffraction limited }} \sim \frac{\lambda}{2}
$$

As a consequence, many attempts have been made to overcome the diffraction limit. Most of them are derived from scanning probe methods: a small object, usually the very end of a sharp tip, is used as a local probe and scanned over the sample surface.
The resolution $f$ of these methods being mainly influenced by the effective probe size $r$ and the decay length $l$ of the interaction, bringing nanometer-sized objects to a very close distance $d$ from the surface allows spatial resolution of the order of 0.1 nm (Eqn. (2)). [1]

$$
f^{\text {local probe }} \propto \sqrt{(r+d) l}
$$

The optical version of these local probe techniques is the scanning near-field optical microscopy (SNOM). ${ }^{[2]}$ By using the end of an optical fiber sharpened to as little as 50 $\mathrm{nm}$, the light spot on the sample is considerably smaller than the diffraction limit. ${ }^{[3]}$ In addition to imaging, this can be used for exciting fluorescence or Raman scattering at the local scale. However, the lateral extent of the illuminated area still prevents a single molecule investigation inside an adsorbed monolayer for instance.

The highest spatial resolution is achieved by the scanning tunneling microscope (STM). ${ }^{[4]}$ An atomically sharp conductive tip is brought very close $(\sim 1 \mathrm{~nm})$ to a conductive sample, and a small bias voltage $V(\mathrm{mV}$ to $\mathrm{V})$ is applied between them (Fig. 1). As a result of the overlap of the tip and surface wavefunctions, a small tunneling current flows (pA to $\mathrm{nA}$ ) in the cap and is used as a feedback signal. Scanning over the surface then results in an extraordinarily sharp image, resolving not only single molecules but even their constituent atoms and 


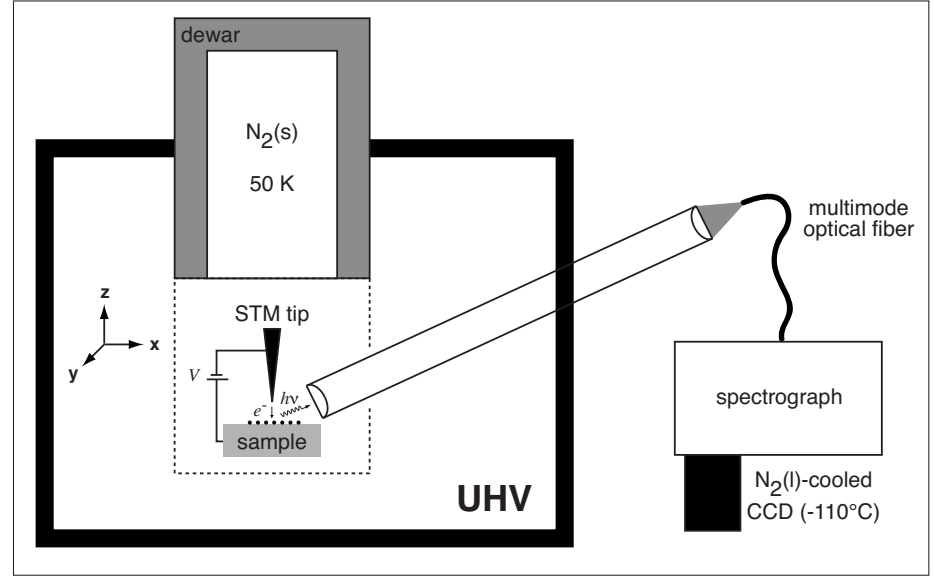

Fig. 1. Experimental setup of the UHV-LT STM induced luminescence spectrometer. The photons generated by the tunneling electrons at lowtemperature and in ultra-high vacuum are collected and guided out of the STM chamber, where an optical fiber guides them to the CCD camera through the spectrograph. (Adapted with permission from ref. [5].)

bonds. ${ }^{[6]}$ However, the tip probes the electronic density at the surface. Therefore, the measured image cannot be directly related to the topography of adsorbed molecules, and the interpretation of the data can be difficult. In particular, the chemical identification of the observed species is not always straightforward.

Being able to combine optical spectroscopy with STM would of course solve this problem. In situ spectroscopic information at the submolecular scale is hence a highly desirable experimental goal, and several directions have been investigated so far. Tipenhanced Raman spectroscopy (TERS), ${ }^{[7,8]}$ where the STM tip is used as a local feature to enhance the Raman intensity by the surface-enhanced Raman spectroscopy (SERS) effect, ${ }^{[9]}$ has been demonstrated recently. ${ }^{[10,11]}$ Large enhancement factors $\left(>10^{-6}\right)$ have been observed for organic molecules on gold, and single-molecule sensitivity has been proven with extremely diluted samples. ${ }^{[12]}$ However, in situ local Raman spectra from individual molecules inside a densely arranged monolayer have still to be observed. STM-tip induced luminescence is another alternative: ${ }^{[13]}$ by using the tunneling electrons to excite electronic levels in the adsorbed species, the luminescence from a single molecule can be detected. For example, an applied bias voltage of $1.5-3 \mathrm{~V}$ results in tunneling electrons with an energy of $1.5-3 \mathrm{eV}(800-400 \mathrm{~nm})$, similar to visible light. And since the tunneling current is highly localized, as proven by high-resolution STM images, STM-induced luminescence has the potential to perform single-molecule spectroscopy.

In order to realize this objective, one should be able to produce a well-defined molecular assembly on a metallic substrate, and to get high-resolution STM images of them. Two main options are possible: room temperature (RT) experiments with the tip immersed in a drop of solvent, and experiments in ultra-high vacuum (UHV) at lowtemperature (LT). The first has the advantage of being relatively simple and cost-effective: the environment of the gap between the tip and the sample is kept free of contaminants by the solvent itself, avoiding the expensive and bulky equipment for high vacuum. The samples are prepared simply by adding a compound in the solution, and if the surface interaction is strong enough, molecules will form self-assembled monolayers (SAM) onto the substrate. ${ }^{[14]}$ Submolecular resolution STM images of various organic samples have been obtained with this method. ${ }^{[15,16]}$ However, the extent of coverage can hardly be controlled, and the SAM evolves with time by desorption/readsorption processes. ${ }^{[17]}$ Furthermore, thermal drift cannot be avoided at RT, resulting in a continuous drift of the scanned area with time. In contrast, sublimation in ultrahigh vacuum (UHV) onto a single crystal is the method of choice, because it offers a complete control of the coverage by varying the time of deposition. ${ }^{[18]}$ In addition, the environment is extremely clean in UHV and the samples remain constant in composition for several days. Working at cryogenic temperatures will then help to immobilize the molecules on the surface. Finally, STM experiments performed in UHV at LT give the best results due to the lack of significant thermal drift.[19]

Under UHV-LT conditions, luminescence due to the decay of localized surface plasmons on metal surfaces has been observed in 1991. ${ }^{[20]}$ More than ten years later, the first successful report of luminescence from supported molecules was published by Ho and coworkers. ${ }^{[21]}$ The key for such measurements is to avoid the quenching of the radiative transitions by the underlying metallic substrate, ${ }^{[22]}$ and they successfully decoupled metal and molecules with a thin insulating layer deposited between them.

However, the observation of several electronic-vibrational transitions is needed for unambiguous chemical identification of complex molecules. Recently, the first energy-resolved fluorescence and phosphorescence spectra of an individual $\mathrm{C}_{60}$ molecule excited by local electron tunneling have been measured in our laboratory. [23] By comparison with the non-local luminescence spectra from $\mathrm{C}_{60}$ dispersed in rare gas and glass matrices, ${ }^{[24]}$ as well as from solid $\mathrm{C}_{60},{ }^{[25]}$ we have been able to identify the observed spectral features with pure electronic and vibronic transitions, thus demonstrating the molecular origin of the detected light.

$\mathrm{C}_{60}$ molecules were deposited on a monocrystalline gold substrate with intermediate layers of sodium chloride in order to decouple the molecules and avoid quenching. $\mathrm{NaCl}$ was evaporated at RT onto $\mathrm{Au}(111),{ }^{[26]}$ and then the $\mathrm{C}_{60}$ molecules were sublimed onto this insulator-covered substrate. Measurements were carried out with a home-built STM working at $50 \mathrm{~K}$ and in UHV, with mechanically cleaved $\mathrm{Pt} /$ Ir tips. A single lens condenser placed inside the vacuum chamber collected the photons emitted from the tunnel junction and guided them to atmospheric pressure, where a second lens refocused the light into a multimode optical fiber (Fig. 1). The signal was then diffracted by a grating spectrograph, and the resulting spectrum was detected by a liquid nitrogen-cooled CCD camera. The spectral resolution of the system was $8 \mathrm{~nm}$ in the energy range of interest (600-900 $\mathrm{nm})$. During the acquisition of the spectra (300 s), the STM tip was kept in tunneling position over a defined position of the sample by a closed feedback loop.

$\mathrm{NaCl}$ forms close-packed islands on $\mathrm{Au}(111)$, with a thickness between one and three monolayers and lateral dimensions up to $1 \mu \mathrm{m}$. $\mathrm{C}_{60}$ molecules deposited onto these ultrathin salt islands aggregate into hexagonal or truncated triangular nanocrystals with a height of several molecular layers, ${ }^{[27]}$ unlike on bare $\mathrm{Au}(111)$ where extended islands are found. ${ }^{[28]}$ Fig. 2a shows a typical STM image of such a sample, with fullerene island grown on bare $\mathrm{Au}(111)$ (A) and on $\mathrm{NaCl}$ islands (B, C and D). The nanocrystals present a minimum height of two layers of $\mathrm{C}_{60}(\mathrm{~B})$. As shown in the high-resolution STM image (Fig. 2b), the fullerene molecules arrange in a hexagonal lattice with an intermolecular distance of $1 \mathrm{~nm}$.

On the bare $\mathrm{Au}(111)$ surface, luminescence is observed at both bias polarities. This is due to the decay of the surface plasmon of gold excited by inelastic electron tunneling (IET), ${ }^{[29]}$ and a similar but atten- 


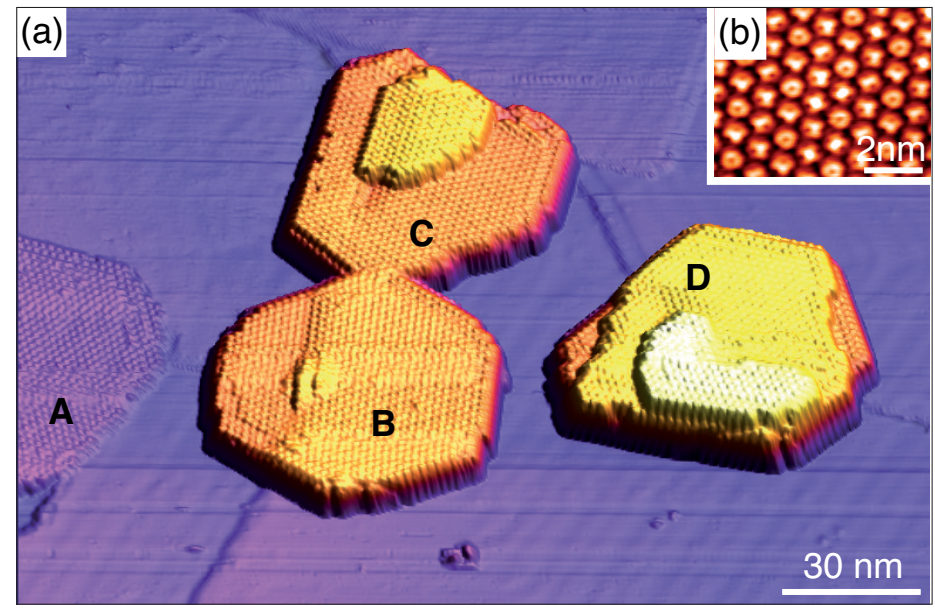

Fig. 2. (a) STM image of $\mathrm{C}_{60}$ nanocrystals on a $\mathrm{NaCl}$ covered $\mathrm{Au}(111)$ substrate. Islands $B, C$ and $D$ consist of up to two, three, and four $C_{60}$ molecular layers, respectively. The area $A$ is a monolayer of $C_{60}$ formed directly on gold (tunneling parameters: $V=-3 \mathrm{~V}, \mathrm{I}=0.02 \mathrm{nA}$ ). (b) Highresolution magnification of the island $\mathrm{B}$. The different bright features (circles and stars) correspond to various adsorption orientations of individual $\mathrm{C}_{60}$ molecules in the monocrystal (tunneling parameters: $\mathrm{V}=-3 \mathrm{~V}, \mathrm{I}=0.1 \mathrm{nA}$ ). (Reproduced with permission from ref. [23]: http://link.aps.org/abstract/ PRL/v95/e196102. Copyright (2005) by the American Physical Society.) (a)

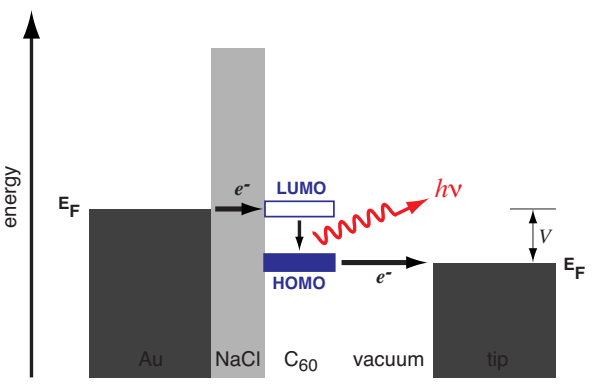

(b)

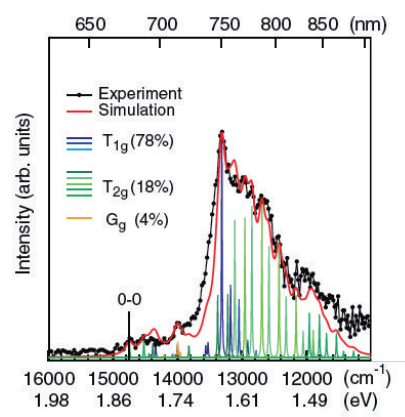

Fig. 3. (a) Energy diagram for $\mathrm{C}_{60}$ deposited on $\mathrm{NaCl}$-covered $\mathrm{Au}(111)$ with a negative bias voltage applied $(\mathrm{V})$. The luminescence is explained by the hot electron injection mechanism. (b) STM-induced fluorescence of a single $\mathrm{C}_{60}$ molecule $(\mathrm{V}=-3 \mathrm{~V}, \mathrm{I}=1 \mathrm{nA})$ and calculated spectrum. (Adapted with permission from ref. [23]. Copyright (2005) by the American Physical Society.) (a)

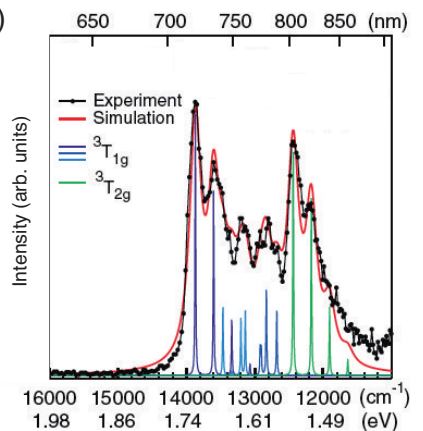

(b)

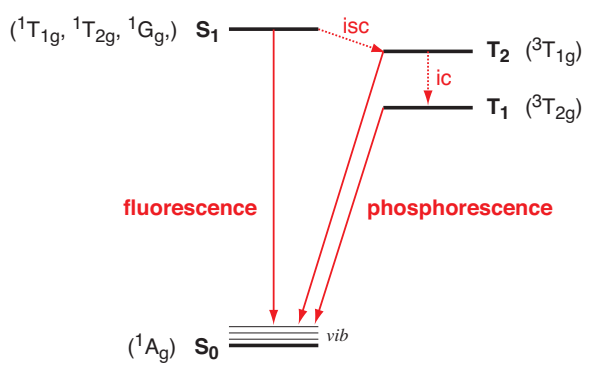

Fig. 4. (a) STM-induced phosphorescence of a single $C_{60}$ molecule $(V=-3 V, I=1 n A)$ along with calculated spectrum and bands assignment. (b) Jablonski diagram of the radiative (solid arrows) and non-radiative (dashed arrows) transitions between electronic states (thick horizontal lines) and vibrational levels (thin horizontal lines). (Adapted with permission from ref. [23]. Copyright (2005) by the American Physical Society.) uated spectrum is observed for $\mathrm{NaCl}-\mathrm{cov}-$ ered $\mathrm{Au}(111)$. In contrast, photons emitted by a single $\mathrm{C}_{60}$ molecule on $\mathrm{NaCl} / \mathrm{Au}(111)$ are excited only by tunneling electrons at negative bias voltages, with a threshold voltage of $V=-2.3 \mathrm{~V}$. This luminescence of $\mathrm{C}_{60}$ is explained by the hot electron injection mechanism (Fig. 3a): at negative bias voltage larger than $-2.3 \mathrm{~V}$, the highest-occupied molecular orbital (HOMO) of $\mathrm{C}_{60}$ is higher in energy than the Fermi level $(\mathrm{EF})$ of the tip. Electrons are hence extracted from the HOMO and tunnel to the tip, while the lowest-unoccupied molecular orbital (LUMO), now lower than the Fermi level of the sample, is populated by electrons tunneling from the substrate. These hot electrons then decay radiatively into the partially empty HOMO, generating the luminescence. Though the inverse mechanism would in principle be possible at positive bias voltage, luminescence is not observed under these conditions. The asymmetry of the HOMO-LUMO gap with respect to EF might be the cause of this absence of luminescence.[30]

Fig. $3 b$ shows the fluorescence of a single $\mathrm{C}_{60}$ molecule excited by local electron tunneling, along with the calculated spectra and the assignment of bands obtained by comparing our results with non-local luminescence and the results of quantum chemical calculations. ${ }^{[24]}$ Although the $\mathrm{S}_{0} \leftarrow \mathrm{S}_{1}$ transition is symmetry forbidden $\left(S_{0}\right.$ and $S_{1}$ have $A_{g}$ and mixed $T_{1 g}, T_{2 g}, G_{g}$ character, respectively), it occurs through HerzbergTeller (HT) and Jahn-Teller (JT) electronvibration coupling. [31] Symmetry lowering in the $\mathrm{C}_{60}$ lattice gives rise to a very weak luminescence signal corresponding to the pure electronic $(0-0) S_{0} \leftarrow S_{1}$ transition at $678 \mathrm{~nm}$. On the other side, the HT vibronically induced $\mathrm{S}_{0} \leftarrow \mathrm{S}_{1}\left(\mathrm{~T}_{1 \mathrm{~g}}, \mathrm{~T}_{2 \mathrm{~g}}, \mathrm{G}_{\mathrm{g}}\right)$ transitions are much more intense $(\lambda>678 \mathrm{~nm})$. In order to quantify the contribution of each symmetry character of $S_{1}$ to the observed fluorescence, we simulated the spectrum using calculated oscillator strengths and experimentally determined frequencies. ${ }^{[24]}$ The ratio of these contributions varies from one to another, differently oriented, $\mathrm{C}_{60}$ molecule in the nanocrystal, reflecting the molecular spatial resolution of the technique. ${ }^{[5]}$ The agreement between measured and calculated spectra indicates that the molecular properties of $\mathrm{C}_{60}$ are preserved in the van der Waals crystal, due to weak interactions between molecules.

Interestingly, phosphorescence of $\mathrm{C}_{60}$ on $\mathrm{NaCl} / \mathrm{Au}(111)$ is also observed (Fig. 4a). The measured spectrum, similar to the luminescence of solid $\mathrm{C}_{60}$ measured by laser spectroscopy, ${ }^{[32]}$ originates from triplet-to-singlet ground state transitions (Fig. 4b). Although symmetry and spin forbidden, an intense pure electronic (0-0) $\mathrm{S}_{0} \leftarrow \mathrm{T}_{1}\left({ }^{3} \mathrm{~T}_{2 \mathrm{~g}}\right)$ transition appears at $803 \mathrm{~nm}$, 
followed by the progression of a JT active mode. At $720 \mathrm{~nm}$, a similarly intense (0-0) $\mathrm{S}_{0} \leftarrow \mathrm{T}_{2}\left({ }^{3} \mathrm{~T}_{1 \mathrm{~g}}\right)$ is observed, followed by a progression of vibronic bands. The energy difference between the two most intense peaks $(0.18 \mathrm{eV})$ corresponds to the splitting of the lowest $\mathrm{C}_{60}$ triplet state, in accordance with the results of electron energy loss spectroscopy (EELS) as well as theoretical calculations. ${ }^{[33,34]}$ Again, as in the case of fluorescence, the measured and calculated spectra are in very good agreement, indicating a single-molecule origin of the emitted light.

Differences in the local environment of the probed $\mathrm{C}_{60}$ molecule may explain the observation of both fluorescence and phosphorescence. However, a spontaneous modification of the tunneling conditions (tip deformation, current instabilities, electric field fluctuations, etc.) cannot completely be ruled out as a potential cause of the preferred deactivation pathway.

In conclusion, the potential of STMinduced luminescence as a local, in situ and spatially highly-resolved spectroscopy has been demonstrated, resulting in unambiguous chemical identification at the single-molecule scale. Nevertheless, being able to measure the pure vibrational spectrum could be a superior technique to probe the local environment of a molecular species. In particular, TERS spectroscopy seems very promising: in principle, all molecules produce a Raman scattering signal, while luminescence is limited to emitting species. However, as the laser beam focused at the tunnel junction in TERS can potentially perturb the STM measuring process (tip instabilities due to local heating, photochemical decomposition or even laser desorption of the sample, etc.), an efficient design of the STM-Raman spectrometer is required to overcome these difficulties. In addition, working at RT with the STM tip immersed in a liquid would be interesting for chemists: the possibility to add several reactants in solution would allow more 'chemical' experiments.

\section{Acknowledgements}

The author would like to express his gratitude to Prof. Wolf-Dieter Schneider from the Ecole Polytechnique Fédérale de Lausanne and to Prof. Neville V. Richardson from the University of St. Andrews for fruitful discussions and comments.

Received: July 27, 2007

[1] G. Binnig, H. Rohrer, Rev. Mod. Phys. 1999, 71, S324.

[2] D. W. Pohl, W. Denk, M. Lanz, Appl. Phys. Lett. 1984, 44, 651.

[3] T. Schmid, T. A. Schmitz, P. D. Setz, B.-S Yeo, W. Zhang, R. Zenobi, Chimia 2006, 60, 783 .
[4] G. Binnig, H. Rohrer, Rev. Mod. Phys. 1987, 59, 615.

[5] E. Cavar, PhD thesis no. 3332, Ecole Polytechnique Fédérale de Lausanne, 2005.

[6] W. Auwärter, A. Weber-Bargioni, A. Riemann, A. Schiffrin, O. Gröning, R. Fasel, J. V. Barth, J. Chem. Phys. 2006, 124, 194708.

[7] M. S. Anderson, Appl. Phys. Lett. 2000, 76, 3130 .

[8] R. M. Stöckle, Y. D. Suh, V. Deckert, R. Zenobi, Chem. Phys. Lett. 2000, 318, 131.

[9] M. Moskovits, Rev. Mod. Phys. 1985, 57, 783.

[10] B. Pettinger, G. Picardi, R. Schuster, G. Ertl, Single Mol. 2002, 3, 285.

[11] C. Vannier, B.-S. Yeo, J. Melanson, R. Zenobi, Rev. Sci. Instrum. 2006, 77, 023104.

[12] W. Zhang, B.-S. Yeo, T. Schmid, R. Zenobi, J. Phys. Chem. C 2007, 111, 1733.

[13] J. K. Gimzewski, B. Reihl, J. H. Coombs, R. R. Schlittler, Z. Phys. B: Condens. Matter 1988, 72, 497.

[14] P. Brodard, H. Fukumura, in 'Advanced Chemistry of Monolayers at Interfaces: Trends in Methodology and Technology', Vol. 14, Ed. T. Imae, Elsevier, 2007, p. 1.

[15] S. Nishio, I.-i. Daizo, H. Matsuda, M. Yoshidome, H. Uji-i, H. Fukumura, Jpn. J. Appl. Phys. 2005, 44/7B, 5417.

[16] H. Uji-i, S. Nishio, H. Fukumura, Chem. Phys. Lett. 2005, 408, 112.

[17] H. Uji-i, M. Yoshidome, J. Hobley, K. Hatanaka, H. Fukumura, Phys. Chem. Chem. Phys. 2003, 5, 4231.

[18] M. Böhringer, K. Morgenstern, W.-D. Schneider, R. Berndt, F. Mauri, A. De Vita, R. Car, Phys. Rev. Lett. 1999, 83, 324.

[19] M.-C. Blüm, E. Cavar, M. Pivetta, F. Patthey, W.-D. Schneider, Angew. Chem. 2005, 117, 5468.

[20] R. Berndt, J. K. Gimzewski, Phys. Rev. Lett. 1991, 67, 3796.

[21] X. H. Qiu, G. V. Nazin, W. Ho, Science 2003, 299, 542.

[22] R. Berndt, R. Gaisch, J. K. Gimzewski, B. Reihl, R. R. Schlittler, W.-D. Schneider, M. Tschudy, Science 1993, 262, 1425.

[23] E. Cavar, M.-C. Blüm, M. Pivetta, F. Patthey, M. Chergui, W.-D. Schneider, Phys. Rev. Lett. 2005, 95, 196102.

[24] A. Sassara, G. Zerza, M. Chergui, F. Negri, G. Orlandi, J. Chem. Phys. 1997, 107, 8731.

[25] D. J. v. d. Heuvel, I. Y. Chan, E. J. J. Groenen, M. Matsushita, J. Schmidt, G. Meijer, Chem. Phys. Lett. 1995, 233, 284.

[26] W. Hebenstreit, M. Schmid, J. Redinger, R. Podloucky, P. Varga, Phys. Rev. Lett. 2000, 85, 5376

[27] Y. Saito, Y. Ishikawa, A. Ohshita, H. Shinohara, H. Nagashima, Phys. Rev. B 1992 , 46, 1846.

[28] E. I. Altman, R. J. Colton, Surf. Sci. 1993, 295, 13.

[29] R. Berndt, J. K. Gimzewski, P. Johansson, Phys. Rev. Lett. 1993, 71, 3493.

[30] R. W. Lof, M. A. v. Veenendaal, B. Ko- opmans, H. T. Jonkman, G. A. Sawatzky, Phys. Rev. Lett. 1992, 68, 3924.

[31] G. Orlandi, F. Negri, Photochem. Photobiol. Sci. 2002, 1, 289.

[32] A. Sassara, G. Zerza, M. Chergui, Chem. Phys. Lett. 1996, 261, 213.

[33] G. Gensterblum, J. J. Pireaux, P. A. Thiry, R. Caudano, J. P. Vigneron, P. Lambin, A. A. Lucas, W. Krätschmer, Phys. Rev. Lett. 1991, 67, 2171.

[34] I. Laszlo, L. Udvardi, J. Mol. Struct: THEOCHEM 1989, 183, 271. 